\title{
Constraining Dark Matter-Baryon Scattering with Linear Cosmology
}

\author{
Cora Dvorkin* and Kfir Blum ${ }^{\dagger}$ \\ Institute for Advanced Study, School of Natural Sciences, Einstein Drive, Princeton, NJ 08540, USA \\ Marc Kamionkowsk团 \\ Department of Physics and Astronomy, Johns Hopkins University, Baltimore, MD 21218, USA
}

\begin{abstract}
We derive constraints on elastic scattering between baryons and dark matter using the cosmic microwave background (CMB) data from the Planck satellite and the Lyman- $\alpha$ forest data from the Sloan Digital Sky Survey. Elastic scattering allows baryons and dark matter to exchange momentum, affecting the dynamics of linear density perturbations in the early Universe. We derive constraints to scattering cross sections of the form $\sigma \propto v^{n}$, allowing for a wide range of velocity dependencies with $-4 \leq n \leq 2$. We improve and correct previous estimates where they exist, including velocityindependent cross section as well as dark matter millicharge and electromagnetic dipole moments. Lyman- $\alpha$ forest data dominates the constraints for $n>-3$, where the improvement over CMB data alone can be several orders of magnitude. Dark matter-baryon scattering cannot affect the halo mass function on mass scales $M>10^{12} M_{\odot}$. Our results imply, model-independently, that a baryon in the halo of a galaxy like our own Milky Way, does not scatter from dark matter particles during the age of the galaxy.
\end{abstract}

\section{Contents}

I. Introduction 2

II. The baryon-dark-matter drag force 3

III. Linear cosmological perturbations with dark-matter-baryon interactions

A. Boltzmann equations 4

B. The momentum-exchange rate coefficient 5

C. Analytic discussion

IV. Numerical results: CMB and Lyman-alpha constraints 7

$\begin{array}{ll}\text { V. Baryon-DM interactions and suppression of small-scale structure } & 9\end{array}$

VI. Late-time effects of baryon-DM interactions 11

VII. Conclusions $\quad[11$

$\begin{array}{lr}\text { Acknowledgments } & 12\end{array}$

A. Thermalization $\quad \lcm{12}$

B. Peculiar velocity beyond leading order $\quad 012$

C. Comparison to previous work and specific dark matter-baryon interaction models

1. Velocity-independent scattering $\quad 13$

2. DM millicharge

3. DM electric and magnetic dipole moment 14

References

*cdvorkin@ias.edu

${ }^{\dagger}$ kblum@ias.edu

${ }^{\ddagger}$ kamion@jhu.edu 


\section{INTRODUCTION}

The canonical dark matter (DM) candidate is assumed to interact with Standard Model particles only gravitationally. The cross section for the simplest weakly-interacting massive particles (WIMPs) [1 [4] to scatter from baryons is non-zero but sufficiently small to be considered effectively zero for scales above a solar mass [5]. However, the shrinking of the canonical-WIMP parameter space from null LHC and direct searches, efforts to explain the coincidence between the DM and baryon densities [6, 7], as well as possible difficulties for collisionless N-body simulations to reproduce observational data [8 13], provide motivation to consider stronger baryon-DM interactions.

In this paper we evaluate the constraints to elastic DM-baryon scattering that arise from the cosmic microwave background (CMB) and large-scale structure (LSS). In the standard scenario of collisionless DM, perturbations to the DM density grow in amplitude early on while the pressure in the baryon-photon fluid prevents it from falling into the DM-dominated potential wells. This dynamics defines the overall shape of the CMB and matter power spectra, and gives rise to characteristic features such as the acoustic peaks seen in the CMB power spectrum and the baryon acoustic oscillations (BAO) in the matter power spectrum. If there is some coupling between DM and baryons, then the drag force between the baryon-photon fluid and the DM affects the baryon-photon oscillations and suppresses the growth of perturbations to the dark matter, and hence total, density. The beautiful agreement between the predictions of the colisionless $\Lambda$ CDM model and the wealth of CMB/LSS data implies that the DM-baryon interaction has to be quite weak, a statement we quantify precisely below.

CMB/LSS constraints to the baryon-DM interaction have been obtained in a variety of previous papers. The first such paper [14] considered velocity-independent scattering and obtained limits from 2dFGRS LSS data and from CMB data from the set of suborbital missions that preceded the Wilkinson Microwave Anisotropy Probe (WMAP). Ref. [15] considered constraints for DM particles that interact with baryons through a DM electromagnetic dipole using early WMAP data. Ref. [16] considered constraints to the baryon-DM interaction from the damping of small-scale structure. DM millicharge have been discussed in [17-21]. Constraints to the baryon-DM interaction from galaxy clusters were presented in Refs. [22, 23]. There is also a body of work on direct detection [24 29], gravitational lensing 30], gas temperature in clusters [31], big-bang nucleosynthesis 32], cosmic rays [33, 34], and cosmic gamma rays [36]. If DM annihilates, then constraints on the Earth's heat flow [35, 36] become important, though this constraint disappears if annihilation is absent as could be the case for asymmetric dark matter [6, 7]. Related studies were addressed in the context of strongly self-interacting dark matter particles, first suggested by Ref. 37].

Our work extends and improves upon previous work on CMB/LSS constraints to the baryon-DM interaction in two ways. First of all, we provide a model-independent analysis, considering cross sections that scale with DMbaryon relative velocity $v$ as $v^{n}$ with arbitrary power-law index $n$. Second, we work out from first principles the effect of scattering in cosmological perturbation theory, highlighting the interplay between bulk velocities and thermal velocities. In prior work it was assumed that the relative baryon-DM bulk velocities are small compared to the thermal velocities. We show that this assumption becomes invalid at redshifts $z \lesssim 10^{4}$, signaling the breakdown of the ordinary linear theory. We introduce an approximation to account for this nonlinearity, and estimate the theoretical uncertainty. We use for our numerical results what we believe to be a conservative estimate of the magnitude of the effects of this nonlinearity. We then use not only the recent Planck data, but also include for the first time in this context constraints to the matter power spectrum from the Lyman-alpha forest measurements. Inclusion of Lyman-alpha data improves, as we will see, upon constraints obtained from the CMB by several orders of magnitude.

The main result of this paper is that a baryon-DM interaction strong enough to affect the global structure of a galaxy like our own Milky Way, through scattering at low redshift, is excluded. As we show, this result is modelindependent, and cannot be circumvented by plausible particle-physics model building, as long as the DM we infer locally by galactic rotation curves and cluster dynamics is the same DM that affects the linear collapse of density perturbations in the early Universe.

This paper is organized as follows. In $₫ \amalg$ we compute the drag force produced by the baryons on the dark-matter fluid due to the DM-baryon interactions. In $₫ \amalg$ we derive the modified Boltzmann equations for the dark matter and the baryons, and provide simple analytical estimates of our results before presenting our numerical constraints in $₫$ IV. In $\$ \mathrm{~V}$ we show the effect of DM-baryon interactions on the halo mass function. Finally, we present our conclusions in $\$$ VI. We derive the heating rate in Appendix $\mathrm{A}$ and give the DM-baryon momentum transfer rate beyond leading order in bulk velocities in Appendix B. In Appendix C we compare our results to previous studies focusing on specific particle-physics models, including velocity-independent cross section as well as dark matter millicharge and electromagnetic dipole moments. 


\section{THE BARYON-DARK-MATTER DRAG FORCE}

Here we calculate the drag force $d \vec{v}_{\chi} / d t$ per unit mass exerted by the baryons on the dark-matter fluid as a consequence of the DM-baryon interaction. Our calculations are valid for $z \lesssim 10^{9}$, where DM particles of mass $m_{\chi} \gtrsim \mathrm{MeV}$ are non-relativistic, and we assume that DM particles and baryons are non-relativistic throughout our analysis.

Consider a DM particle of velocity $\vec{v}_{\chi}$, moving in a background of non-relativistic baryons with thermal velocity distribution $f_{b}\left(v_{b}\right)$ as a function of baryon velocity $v_{b}$, in a frame where the baryon distribution is isotropic. In this frame, the baryon velocity distribution depends only on the magnitude $v_{b}$ of the velocity, not on its direction, and momentum exchange with baryons drives the DM velocity towards zero. The change in DM momentum per collision is

$$
\Delta \vec{p}_{\chi}=\frac{m_{\chi} m_{b}}{m_{\chi}+m_{b}}\left|\vec{v}_{\chi}-\vec{v}_{b}\right|\left(\hat{n}-\frac{\vec{v}_{\chi}-\vec{v}_{b}}{\left|\vec{v}_{\chi}-\vec{v}_{b}\right|}\right)
$$

to leading order in velocities, where $\hat{n}$ is the direction of the scattered DM particle in the center-of-mass frame and $m_{b}$ and $m_{\chi}$ respectively the baryon and dark-matter masses. The acceleration experienced by the DM is then

$$
\begin{aligned}
\frac{d \vec{v}_{\chi}}{d t} & =\frac{\rho_{b}}{m_{\chi}+m_{b}} \int d v_{b} v_{b}^{2} f_{b}\left(v_{b}\right) \int \frac{d \hat{n}_{b}}{4 \pi} \int d \hat{n}\left(\frac{d \sigma\left(\left|\vec{v}_{\chi}-\vec{v}_{b}\right|\right)}{d \hat{n}}\right)\left|\vec{v}_{\chi}-\vec{v}_{b}\right|^{2}\left(\hat{n}-\frac{\vec{v}_{\chi}-\vec{v}_{b}}{\left|\vec{v}_{\chi}-\vec{v}_{b}\right|}\right) \\
& =-\frac{\rho_{b} \vec{v}_{\chi}}{m_{\chi}+m_{b}} \frac{v_{\chi}^{4}}{2} \int_{0}^{\infty} d x x^{2} f_{b}\left(x v_{\chi}\right) \int_{-1}^{1} d y \bar{\sigma}\left(v_{\chi} \sqrt{1+x^{2}-2 x y}\right) \sqrt{1+x^{2}-2 x y}(1-x y),
\end{aligned}
$$

where $\rho_{b}$ is the baryon mass density. Here $d \sigma(v) / d \hat{n}$ is the differential cross section for baryon-DM scattering as a function of the baryon-DM relative velocity $v$. We also define the momentum-transfer cross section,

$$
\bar{\sigma}(v) \equiv \int d c_{\theta}\left(1-c_{\theta}\right)\left(\frac{d \sigma(v)}{d c_{\theta}}\right)
$$

where $\theta$ is the center-of-mass scattering angle, $c_{\theta}=\cos \theta$.

We take the cross section $\bar{\sigma}(v)$ to have a power-law dependence on baryon-DM relative velocity $v$ (where the speed of light is $c=1$ ),

$$
\bar{\sigma}(v)=\sigma_{0} v^{n} .
$$

We work out the momentum exchange for arbitrary $n$, and present results for selected values. Note that, e.g., $n=-1$ comes about from a Yukawa potential (massive-boson exchange), $n=-2$ occurs if DM has an electric dipole moment [15], and $n=-4$ occurs for DM millicharge [17 20]. We take $\bar{\sigma}$ here to be the scattering cross section for DM from hydrogen, and then correct it below to account for the additional scattering from helium.

In the early Universe, the baryon velocity distribution (in the isotropic frame) is

$$
f_{b}\left(v_{b}\right)=\sqrt{\frac{2}{\pi}} \frac{1}{u_{b}^{3}} e^{-\left(v_{b} / u_{b}\right)^{2} / 2}
$$

with $u_{b}^{2}=T_{b} / m_{b}$. For the DM we assume a similar Maxwell distribution,

$$
f_{\chi}\left(v_{\chi}\right)=\sqrt{\frac{2}{\pi}} \frac{1}{u_{\chi}^{3}} \exp \left[-\frac{\left(\hat{n}_{\chi} v_{\chi}-\vec{V}_{\chi}\right)^{2}}{2 u_{\chi}^{2}}\right],
$$

with $u_{\chi}^{2}=T_{\chi} / m_{\chi}$, boosted with peculiar velocity $\vec{V}_{\chi}$ with respect to the baryon frame. The rate of change of the peculiar velocity is ${ }^{1}$

$$
\frac{d \vec{V}_{\chi}}{d t}=\int \frac{d \hat{n}_{\chi}}{4 \pi} \int d v_{\chi} v_{\chi}^{2} f_{\chi}\left(v_{\chi}\right) \frac{d \vec{v}_{\chi}}{d t}
$$

\footnotetext{
1 The subscript $\chi$ on $d \vec{V}_{\chi} / d t$ is there to emphasize that the deceleration of the DM fluid is different than that of the baryons, due to the difference in inertia of the two fluids, even though the instantaneous relative velocity is of course the same as measured in either the isotropic DM or baryon frames.
} 
In general, there are two velocity scales that enter $d \vec{V}_{\chi} / d t$. The first is the thermal velocity dispersion,

$$
\left\langle(\Delta \vec{v})^{2}\right\rangle=\left\langle\left(\vec{v}_{\chi}-\vec{v}_{b}\right)^{2}\right\rangle=3\left(\frac{T_{b}}{m_{b}}+\frac{T_{\chi}}{m_{\chi}}\right),
$$

where $\langle\ldots\rangle$ denotes thermal average. The second is the peculiar velocity $V_{\chi}$ itself. In the limit where the peculiar velocity is smaller than the velocity dispersion, $V_{\chi}^{2}\left\langle\left\langle(\Delta \vec{v})^{2}\right\rangle\right.$, we find

$$
\frac{d \vec{V}_{\chi}}{d t}=-\vec{V}_{\chi} \frac{c_{n} \rho_{b} \sigma_{0}\left(\frac{\left\langle(\Delta \vec{v})^{2}\right\rangle}{3}\right)^{\frac{n+1}{2}}}{m_{\chi}+m_{b}}
$$

at leading order in $\left(V_{\chi}^{2} /\left\langle(\Delta \vec{v})^{2}\right\rangle\right)$, with

$$
c_{n}=\frac{2^{\frac{n+5}{2}} \Gamma\left(3+\frac{n}{2}\right)}{3 \sqrt{\pi}}
$$

evaluating to $c_{n} \approx\{0.27,0.33,0.53,1,2.1,5,13,35,102\}$ for $n=\{-4,-3,-2,-1,0,1,2,3,4\}$.

In the limit that the peculiar velocity is larger than the velocity dispersion, the calculation reduces to the deceleration of the relative motion between two cold flows. The deceleration of the DM fluid in this case is given by

$$
\frac{d \vec{V}_{\chi}}{d t}=-\vec{V}_{\chi} \frac{\rho_{b} \sigma_{0}\left|V_{\chi}\right|^{n+1}}{m_{\chi}+m_{b}}
$$

at leading order in $\left(\left\langle(\Delta \vec{v})^{2}\right\rangle / V_{\chi}^{2}\right)$.

Note that in general, the dependence of $d V_{\chi} / d t$, the drag force per unit mass, on the baryon-DM relative velocity is not linear. In the limit $V_{\chi}^{2} \ll\left\langle(\Delta \vec{v})^{2}\right\rangle$, the dependence reduces to linear. In the opposite limit, $V_{\chi}^{2} \gg\left\langle\Delta \vec{v}^{2}\right\rangle$, the dependence on $V_{\chi}$ is nonlinear unless $n=-1$. In the early Universe, as we look further backwards in time, there comes a time when typical peculiar velocities become small in comparison to the thermal velocity dispersion. The transition occurs around redshift $z \sim 10^{4}$ (see Fig. 1). At earlier times (higher redshift), Eq. (9) then tell us that we may use linear perturbation theory in order to calculate the evolution of the peculiar velocity $\vec{V}_{\chi}$. In what follows, we use this observation to calculate precisely the evolution of modes at high redshift in order to compare with cosmological data. We discuss later on the complication arising at $z<10^{4}$, where the problem becomes nonlinear.

\section{LINEAR COSMOLOGICAL PERTURBATIONS WITH DARK-MATTER-BARYON INTERACTIONS}

\section{A. Boltzmann equations}

We now consider the modifications to the Boltzmann equations for dark matter and baryons that arise from the baryon-DM coupling. We work in synchronous gauge, following the notation and conventions of Ref. [38]. We allow for a nonzero peculiar velocity for DM that arises from the interaction with baryons [14, 15] and defined so that the DM peculiar velocity vanishes in the absence of scattering. The evolution equations for the DM and baryon density fluctuations, $\delta_{\chi}$ and $\delta_{b}$ respectively, and velocity divergence, $\theta_{\chi}$ and $\theta_{b}$, respectively, are given for a Fourier mode of wavenumber $k$ by

$$
\begin{aligned}
& \dot{\delta_{\chi}}=-\theta_{\chi}-\frac{\dot{h}}{2}, \quad \dot{\delta_{b}}=-\theta_{b}-\frac{\dot{h}}{2}, \\
& \dot{\theta_{\chi}}=-\frac{\dot{a}}{a} \theta_{\chi}+c_{\chi}^{2} k^{2} \delta_{\chi}+R_{\chi}\left(\theta_{b}-\theta_{\chi}\right), \\
& \dot{\theta_{b}}=-\frac{\dot{a}}{a} \theta_{b}+c_{b}^{2} k^{2} \delta_{b}+R_{\gamma}\left(\theta_{\gamma}-\theta_{b}\right)+\frac{\rho_{\chi}}{\rho_{b}} R_{\chi}\left(\theta_{\chi}-\theta_{b}\right),
\end{aligned}
$$


where $\rho_{\chi}\left(\rho_{b}\right)$ is the DM (baryon) mass density, and an overdot denotes derivative with respect to conformal time. We derive the DM-baryon momentum-exchange coefficient $R_{\chi}$ below in Sec. IIIB.

The DM and baryon temperatures evolve according to

$$
\begin{aligned}
\dot{T}_{\chi}= & -2 \frac{\dot{a}}{a} T_{\chi}+\frac{2 m_{\chi}}{m_{\chi}+m_{\mathrm{H}}} R_{\chi}^{\prime}\left(T_{b}-T_{\chi}\right), \\
\dot{T}_{b}= & -2 \frac{\dot{a}}{a} T_{b}+\frac{2 \mu_{b}}{m_{\chi}+m_{\mathrm{H}}} \frac{\rho_{\chi}}{\rho_{b}} R_{\chi}^{\prime}\left(T_{\chi}-T_{b}\right) \\
& +\frac{2 \mu_{b}}{m_{e}} R_{\gamma}\left(T_{\gamma}-T_{b}\right) .
\end{aligned}
$$

Here, $\mu_{b} \simeq m_{\mathrm{H}}\left(n_{H}+4 n_{\mathrm{He}}\right) /\left(n_{H}+n_{\mathrm{He}}+n_{e}\right)$ is the mean molecular weight for the baryons, and $R_{\gamma}=$ $(4 / 3)\left(\rho_{\gamma} / \rho_{b}\right) a n_{e} \sigma_{T}$ is the usual Compton collision term [38]. The thermalization rate $R_{\chi}^{\prime}$ is related to the momentum exchange rate $R_{\chi}$ (with $R_{\chi}^{\prime} \rightarrow R_{\chi}$ in the heavy DM limit) and is given in Sec. II B below.

Our calculations apply to cold DM with mass $m_{\chi}>\mathrm{MeV}$, that is non-relativistic at redshift $z<10^{9}$. We therefore neglect possible direct momentum transfer between the photon and DM fluids, and consider only direct interaction with baryons. For the calculations we will be interested in, the DM sound speed $c_{\chi}^{2}$ is unimportant, and we neglect the corresponding term in what follows.

\section{B. The momentum-exchange rate coefficient}

If the peculiar velocity is small compared with the thermal velocity-i.e., if $V_{\chi}^{2} \ll\left\langle(\Delta \vec{v})^{2}\right\rangle$ - then the DM-baryon momentum-exchange and thermalization rate coefficients, appearing in Eqs. (12) and (13), can be read from Eqs. (9) and (A2) to be

$$
R_{\chi}=\frac{a c_{n} \rho_{b} \sigma_{0}}{m_{\chi}+m_{\mathrm{H}}}\left(\frac{T_{b}}{m_{\mathrm{H}}}+\frac{T_{\chi}}{m_{\chi}}\right)^{\frac{n+1}{2}} \mathcal{F}_{\mathrm{He}}
$$

and

$$
R_{\chi}^{\prime}=R_{\chi}\left[1+\frac{3 m_{\mathrm{H}}}{m_{\chi}+4 m_{\mathrm{H}}}\left(\frac{1-f_{\mathrm{He}}}{\mathcal{F}_{\mathrm{He}}}-1\right)\right],
$$

respectively, with $R_{\chi}^{\prime} \simeq R_{\chi}$ for heavy DM.

We include a correction factor,

$$
\begin{aligned}
\mathcal{F}_{\mathrm{He}} & =1-f_{\mathrm{He}}+f_{\mathrm{He}} \frac{\sigma_{\mathrm{He}}}{\sigma_{0}} \frac{1+\frac{m_{\mathrm{H}}}{m_{\chi}}}{1+\frac{4 m_{\mathrm{H}}}{m_{\chi}}}\left(\frac{1+\frac{T_{\chi} m_{\mathrm{H}}}{T_{b} m_{\chi}}}{1+\frac{4 T_{\chi} m_{\mathrm{H}}}{T_{b} m_{\chi}}}\right)^{\frac{n+1}{2}} \\
& \simeq 1+0.24\left(\frac{\sigma_{\mathrm{He}}}{\sigma_{0}}-1\right),
\end{aligned}
$$

for scattering from helium with mass $m_{\mathrm{He}} \simeq 4 m_{\mathrm{H}}$ and mass fraction $f_{\mathrm{He}} \simeq 0.24$. The approximation on the second line of Eq. (16) is applicable if the DM is heavier than helium. The value of $\mathcal{F}_{\mathrm{He}}$ depends on the ratio $\left(\sigma_{\mathrm{He}} / \sigma_{0}\right)$ between the cross section for scattering on helium to that for scattering on hydrogen. Plausible numerical values are, e.g., $\mathcal{F}_{\mathrm{He}}=4.6$ or $\mathcal{F}_{\mathrm{He}}=1.7$, valid for DM mass above a few $\mathrm{GeV}$ with the same amplitude for scattering from protons and neutrons and, respectively, coherent or incoherent scattering on helium. Nevertheless, as $\mathcal{F}_{\mathrm{He}}$ involves some model dependence, in reporting our numerical results we conservatively set $\left(\sigma_{\mathrm{He}} / \sigma_{0}\right)=0$, fixing $\mathcal{F}_{\mathrm{He}}=0.76$ unless explicitly stated otherwise.

For $V_{\chi}^{2} \ll\left\langle(\Delta \vec{v})^{2}\right\rangle$, the coefficient $R_{\chi}$ is independent of $\theta_{\chi}-\theta_{b}$, and the DM-baryon drag that appears in Eq. (12) is linear in the velocity perturbation. The usual linear-theory approach, obtained by solving the linearized Boltzmann equations independently for each Fourier mode, is valid.

However, this assumption $\left(V_{\chi}^{2} \ll\left\langle(\Delta \vec{v})^{2}\right\rangle\right)$ is not always valid. The rms DM-baryon relative velocity is given by 39.

$$
V_{\mathrm{RMS}}^{2}=\left\langle\vec{V}_{\chi}^{2}\right\rangle_{\xi}=\int \frac{d k}{k} \Delta_{\xi}\left(\frac{\theta_{b}-\theta_{c}}{k}\right)^{2}
$$




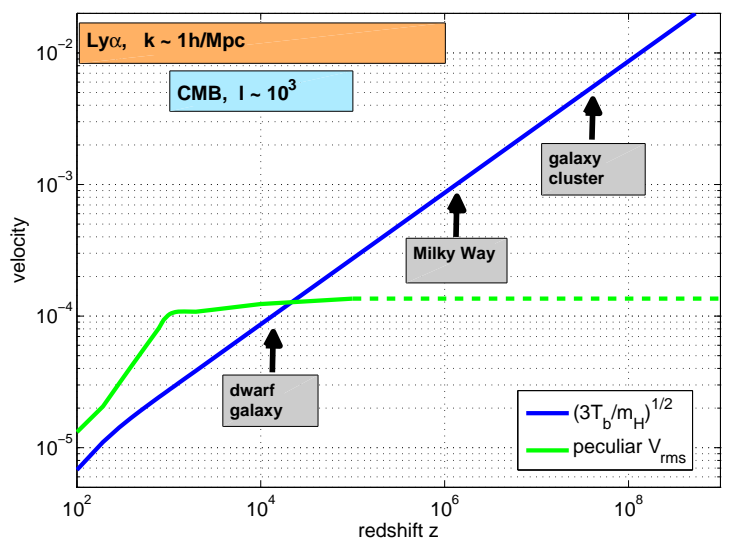

FIG. 1: Cosmological proton thermal velocity (blue), and peculiar baryon-DM relative velocity (green). The redshifts probed by CMB and Lyman- $\alpha$ forest measurements are roughly marked by blue and orange boxes, respectively.

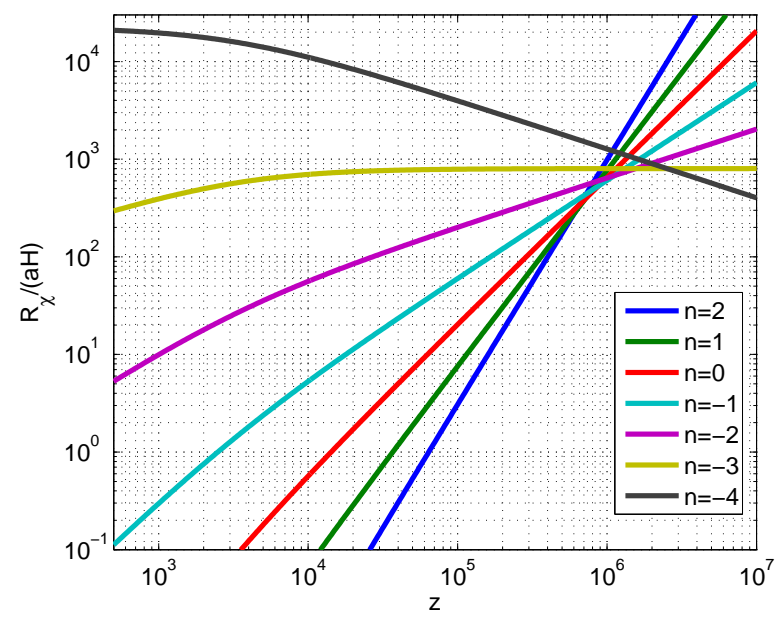

FIG. 2: Momentum-transfer rate $R_{\chi}$ vs. redshift for different values of $n$ between -4 to +2 . All of the curves are normalized to satisfy a mean free path of $\sim 0.5 \mathrm{Mpc}$ for proton scattering on DM at the MW solar cycle; see Eq. (25) and the discussion around it.

where $\langle\ldots\rangle_{\xi}$ denotes an average with respect to the primordial curvature perturbation and $\Delta_{\xi} \simeq 2.4 \times 10^{-9}$ is the primordial curvature variance per $\log k$. The value of $V_{\mathrm{RMS}}$ is shown as the green curve in Fig. 1 (for $z>10^{5}$, we replace the direct calculation of $V_{\mathrm{RMS}}$ by analytic estimate). The peculiar velocity becomes larger than the baryon thermal velocity (the blue curve) below $z \sim 10^{4}$. At later times (lower redshift), the effect of baryon-DM scattering will have a nonlinear dependence on peculiar velocity, as discussed at the end of Section II. This implies that the drag terms in the Boltzmann equations for $\theta_{b}$ and $\theta_{c}$ are no longer linear in $\theta_{b}$ and $\theta_{c}$, mixing together the evolution of different Fourier modes.

To improve the domain of validity of linear theory, we extend the rate coefficient of Eq. (14) by summing together the thermal and peculiar rms velocity dispersion,

$$
R_{\chi} \rightarrow \frac{a \rho_{b} \sigma_{0} \mathcal{F}_{\mathrm{He}}}{m_{\chi}+m_{\mathrm{H}}} c_{n}\left(\frac{T_{b}}{m_{\mathrm{H}}}+\frac{T_{\chi}}{m_{\chi}}+\frac{V_{\mathrm{RMS}}^{2}}{3}\right)^{\frac{n+1}{2}} .
$$

This "mean-field" approach is generally valid at $z>10^{4}$, but should also apply at later times for modes with wavelengths $k \lesssim 0.1 \mathrm{Mpc}^{-1}$, long compared with those that contribute most to the rms peculiar velocity at $z<10^{4}$. It also obtains the correct parametric scaling for short-wavelength modes at low redshift, though the numerical coefficient $c_{n}$, encapsulating thermal velocity integrals, needs to be modified in this limit.

As we show below, models with $n \geq-2$ are strongly constrained by LSS data for which the relevant dynamics 
occurs at relatively high redshift, $z \gg 10^{4}$, where Eq. (14) (and similarly, in this regime, Eq. (18)) is reliable. For all such models $(n \geq-2)$, our constraints derived using Eq. (18) are directly applicable. Models with scattering cross section that increases rapidly at decreasing velocity, $n \leq-3$, are less constrained by LSS and, instead, more strongly constrained by CMB data, that is sensitive to perturbation evolution at $z<10^{4}$. For such models $(n \leq-3)$, using Eq. (18) rather than Eq. (14) makes a significant difference. This means that our linear calculation is less reliable, and that nonlinear (and thus non-gaussian) effects cannot be neglected. Nevertheless, note from Eq. (10) that for models with $n \leq-3$ the coefficient $c_{n}$ is smaller than unity. Comparing to Eq. (11), describing the momentum transfer in the cold-flow limit, we learn that our use of Eq. (18) is likely conservative, meaning that a more detailed treatment of the nonlinear $z<10^{4}$ regime for these models would most likely yield even stronger constraints.

We conclude that for our practical purpose of obtaining conservative limits on the DM-baryon interactions, Eq. (18) is adequate for deriving model-independent results, and we use it in the bulk of our analysis. For completeness, when quoting our numerical results (see Sec. IV]), we also report results using Eq. (14) instead of (18). We leave a precise treatment of the nonlinear effects induced by DM-baryon couplings, as well as the resulting secondary non-Gaussianity, for future work.

In our numerical analysis, we modify CAMB to include the new perturbation equations above. In what follows we describe the analytic behavior of the solution before moving on to the results.

\section{Analytic discussion}

Before moving to the numerical results, we provide some simple analytic estimates. We focus on redshifts $z>300$ when the baryon temperature follows the CMB temperature, $T_{b} \simeq T_{\gamma}$, due to Thomson scattering. Since the dark matter does not scatter from photons, we expect that $T_{\chi}<T_{b}$. Then, if $m_{\chi}>m_{b}$, we can for simple estimates neglect $T_{\chi} / m_{\chi}$ relative to $T_{b} / m_{b}$. Comparing the momentum exchange rate $R_{\chi}$ to the comoving Hubble expansion rate $a H=(\dot{a} / a)$, we have

$$
\begin{aligned}
\frac{R_{\chi}}{a H}= & \frac{c_{n} \rho_{b} \sigma_{0}}{H m_{\chi}}\left(\frac{T_{b}}{m_{\mathrm{H}}}\right)^{\frac{n+1}{2}} \mathcal{F}_{\mathrm{He}} \\
\simeq & 10 c_{n} \frac{\sigma_{0} / m_{\chi}}{\mathrm{cm}^{2} / \mathrm{g}} \frac{H\left(z=10^{5}\right)}{H(z)}\left(\frac{z}{10^{5}}\right)^{3.5} \\
& \times\left[1.6 \cdot 10^{-4}\left(\frac{z}{10^{5}}\right)^{\frac{1}{2}}\right]^{n} \mathcal{F}_{\mathrm{He}} .
\end{aligned}
$$

At high redshift $z>10^{4}$, Eq. (19) can be directly translated to a model-independent constraint on the interaction between DM and baryons in a contemporary $(z=0)$ system like our own Milky Way (MW) galaxy. To see this, note that if we choose $\sigma_{0}$ for the different values of $n$ so that $\bar{\sigma}(v)$ is the same at the velocity $v_{\mathrm{MW}}=10^{-3}$, characteristic of the virial velocity in a MW-type halo, then the scattering rates for all $n$ will coincide, up to the $\mathcal{O}(1)$ coefficients $c_{n}$ of Eq. (10), at a common redshift $z$ where the thermal velocity is approximately equal to $v_{\mathrm{MW}}$, as in Fig. 10. We illustrate this behavior in Fig. 22 where we normalize $\sigma_{0}$ so that the scattering rate for all $n$ will yield a baryon-DM mean free path of about $1 \mathrm{Mpc}$ in our MW galaxy. We learn that with this normalization, for all values of $n$, the rate of momentum exchange between DM and baryons is much faster than expansion for $z>10^{5}$. Comparing to the reach of CMB and Lyman- $\alpha$ observables, which are sensitive to the evolution of linear perturbations at these redshifts, we expect that linear cosmology places strong constraints on baryon-DM scattering for any velocity dependence, implying mean free path $\lambda \gg 1 \mathrm{Mpc}$, orders of magnitude larger than the $\mathcal{O}(10 \mathrm{kpc})$ scale size of the galaxy itself.

\section{NUMERICAL RESULTS: CMB AND LYMAN-ALPHA CONSTRAINTS}

We incorporate the parameter $\sigma_{0}$ into a Markov Chain Monte Carlo (MCMC) likelihood analysis ${ }^{2}$ [40] of Planck data [41] and measurements of the Lyman- $\alpha$ flux power spectrum from the Sloan Digital Sky Survey [42]. We checked that adding ACT [43] and SPT [44] data to the CMB analysis makes only a small improvement to the results. We run the MCMC to determine $95 \%$ confidence level (CL) constraints on $\sigma_{0}$, fixing the value of $m_{\chi}$ and of the power-law index $n$ in each run.

\footnotetext{
2 http://cosmologist.info/cosmomc/
} 
Having obtained a constraint on $\sigma_{0}$ in this way for $m_{\chi}=10 \mathrm{GeV}$, we present our result as a constraint on $\left(\sigma_{0} / m_{\chi}\right)$, valid for any value of $m_{\chi}$ subject to $m_{\chi} \gg m_{\mathrm{H}}$, and quoted separately for different values of $n$. Note that, in the limit of $m_{\chi} \gg m_{\mathrm{H}}$, there is no dependence on $n$ in the scaling of the bound as function of $m_{\chi}$ for fixed $\sigma_{0}$, to leading order in $\left(m_{\mathrm{H}} / m_{\chi}\right)$. This is so because all the dynamical difference between the models is contained in the velocity dependence, where the thermal dispersion becomes dominated by the baryons, $\left\langle(\Delta \vec{v})^{2}\right\rangle \approx 3\left(T_{b} / m_{b}\right)$ to leading order in $\left(m_{\mathrm{H}} / m_{\chi}\right)$. While we do not discuss here in detail the limit $m_{\chi}<m_{\mathrm{H}}$, we note that the set of equations presented in Sec. III provides all of the information required to evaluate the bounds in the low- $m_{\chi}$ limit, as long as the DM is non-relativistic throughout the time of interest $z \lesssim 10^{9}$ (satisfied for $m_{\chi} \gtrsim 1 \mathrm{MeV}$ ).

We determine joint constraints on $\sigma_{0}$ and the basic set of $\Lambda \mathrm{CDM}$ cosmological parameters,

$$
p_{\mu}=\left\{\Omega_{b} h^{2}, \Omega_{\chi} h^{2}, \tau, \theta, A_{s}, n_{s}\right\} .
$$

Here $\Omega_{b} h^{2}$ is the physical baryon density, $\Omega_{\chi} h^{2}$ is the physical dark matter density, $\tau$ is the reionization optical depth, and $\theta$ is the angular size of the sound horizon at recombination. We ignore tensor modes and assume a flat geometry.

Our numerical results are summarized in Table [. In obtaining these bounds, instead of solving for $T_{\chi}$ [which can easily be done using Eq. [13)] we simply set $T_{\chi}=T_{b}$. The induced error is of $\mathcal{O}\left(m_{\mathrm{H}} / m_{\chi}\right)$ for heavy DM.

\begin{tabular}{||c||c|c|c||}
\hline$n$ & CMB $\left(95 \% \mathrm{CL}, \mathrm{cm}^{2} / \mathrm{g}\right)$ & CMB + Lyman- $\alpha\left(95 \% \mathrm{CL}, \mathrm{cm}^{2} / \mathrm{g}\right)$ & $\lambda(\mathrm{MW})$ \\
\hline \hline-4 & $1.8 \times 10^{-17}$ & $1.7 \times 10^{-17}$ & $27 \mathrm{Gpc}$ \\
\hline-2 & $3.0 \times 10^{-9}$ & $6.2 \times 10^{-10}$ & $738 \mathrm{Mpc}$ \\
\hline-1 & $1.6 \times 10^{-5}$ & $1.4 \times 10^{-6}$ & $313 \mathrm{Mpc}$ \\
\hline 0 & 0.12 & $3.3 \times 10^{-3}$ & $138 \mathrm{Mpc}$ \\
\hline+2 & $1.3 \times 10^{5}$ & $9.5 \times 10^{3}$ & $46 \mathrm{Mpc}$ \\
\hline
\end{tabular}

TABLE I: 95\%CL constraints on $\left(\sigma_{0} / m_{\chi}\right)$ from CMB alone (with Planck data) and from CMB in combination with Lyman- $\alpha$ data from the SDSS. Results are valid for $m_{\chi} \gg m_{\mathrm{H}}$, and conservatively neglect scattering from helium, setting $\mathcal{F}_{\mathrm{He}}=0.76$ (adding coherent isospin-independent scattering on helium would tighten the bounds by a factor of 6). First column: powerlaw index $n$ of Eq. (4). Second column: CMB alone, constraint in units $\mathrm{of}_{\mathrm{cm}}^{2} / \mathrm{g}$. Third column: combined CMB and Lyman- $\alpha$. Fourth column: minimal mean free path for baryon scattering on DM in the MW solar cycle $\left(\rho_{\chi} \sim 0.4 \mathrm{GeV} / \mathrm{cm}^{3}\right.$, $v=v_{\mathrm{MW}} \sim 10^{-3}$ ), using the CMB + Lyman- $\alpha$ constraint.

These constraints are obtained using the momentum-transfer rate given in Eq. (18). As discussed at the end of Sec. III B at redshift $z<10^{4}$ Eq. (18) provides only an approximate treatment of the perturbation equations as the full evolution becomes nonlinear ${ }^{3}$. To estimate the impact of our approximation, we compare the constraints reported in Tab. (1 to the constraints obtained using Eq. (14), instead of (18). For the the $n=-2, n=0$, and $n=+2$ models, we find that the CMB+Lyman- $\alpha$ constraints exhibit essentially no change. This happens because for these models, Lyman- $\alpha$ dominates the constraint, and the matter power spectrum on the scales probed by Lyman- $\alpha$ is determined by mode evolution at $z \gg 10^{4}$, where Eqs. (14) and (18) are equally valid. In contrast, the model with $n=-4$ is constrained primarily by the CMB data, and is sensitive to the appearance of $V_{\mathrm{RMS}}^{2}$ in Eq. (18) that regularizes an otherwise decreasing thermal velocity. Using Eq. (14) instead of (18) for the model with $n=-4$, we would find an artificially stronger bound, $\left(\sigma_{0} / m_{\chi}\right)<1.4 \times 10^{-18}$, more constraining by a factor of 10 compared with the number we quote in Tab. [ We believe that our simplified analysis of the $n=-4$ case in the nonlinear regime is conservative, and leaves room for significant improvement of the constraints. This could be of particular interest as $n=-4$ arises in simple particle physics models where DM has a small electric charge.

In Fig. 3 we show the effect of DM-baryon scattering on the CMB and matter power spectra, using for the plots the 95\% CL limits from the CMB + Lyman- $\alpha$ chains, taken from Tab. I We add in Fig. 3 (right panel) the experimental Lyman- $\alpha$ data point used in the likelihood analysis, at $k=1.03 \mathrm{~h} / \mathrm{Mpc}$, showing the $95 \%$ CL limit of both amplitude and slope. In the CMB plot, we denote the $\pm 1 \sigma$ error bars of Planck, including beam noise and cosmic variance, as black $(+)$ marks.

In Fig. 4 we show separately the slope of the linear matter power spectra for the different models, along with the experimental value and its 95\%CL limit coming from the Lyman- $\alpha$ analysis done in Ref. [42].

Finally, we comment that the likelihood procedure given in Ref. [42] strictly applies only to cosmological models with a power-law matter power spectrum. This assumption is not completely satisfied in our framework, where a large

\footnotetext{
3 This issue is relevant for models with $n \neq-1$. For $n=-1$, Eqs. 14 and 18 coincide.
} 

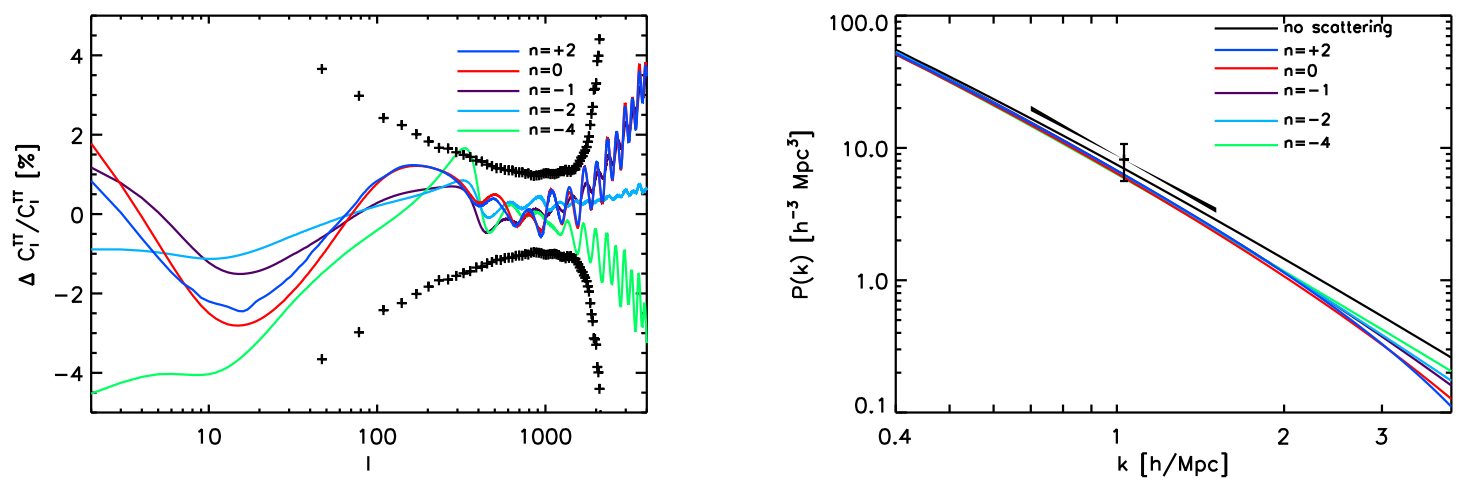

FIG. 3: Left panel: Relative difference of the CMB power spectra of models with different velocity-dependent cross sections to the best fit $\Lambda$ CDM model. The cross sections of the different models correspond to the 95\%CL limit from the CMB + Lyman- $\alpha$ analysis (see Tab. I), while all other cosmological parameters are taken to optimize the likelihood for this given cross section. Right panel: Matter power spectra at $z=3$. The data point corresponds to the linear theory best fit amplitude using Lyman- $\alpha$ data from [42]. The error bar corresponds to the 95\% CL limit on the amplitude. The black band denotes the range of linear matter power spectra slopes allowed at the $95 \%$ CL limit at $k=1.03 \mathrm{~h} / \mathrm{Mpc}$.

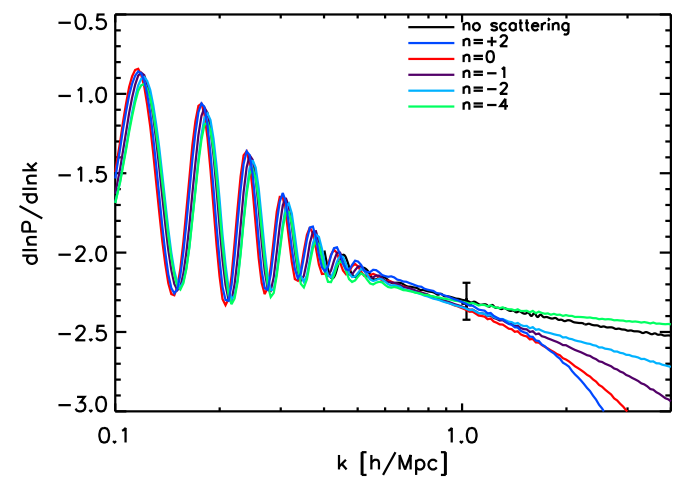

FIG. 4: Slope $d \ln P / d \ln k$ for the different models, as a function of wave number. The data point corresponds to the best fit value of the linear matter power spectrum slope from the Lyman- $\alpha$ measurement in Ref. [42], and the error bar on the point corresponds to the $95 \%$ CL limit.

scattering cross section (for models with $n>-4$ ) would cause a cutoff in the matter power spectrum on small scales (large $k$ ). In practice, as evident in Fig. 3, the Lyman- $\alpha$ data is restrictive enough to render the power spectra of our models, where they are not overwhelmingly excluded, sufficiently close in form to a simple power law in the range of $k=\mathcal{O}\left(1 \mathrm{Mpc}^{-1}\right)$, where reliable data currently exists. This statement holds true for $n$ that are not too largely positive, in which case the cutoff develops quickly as a function of $k$; our model with $n=+2$ provides a marginal example for this situation. For such models with large positive velocity dependence, including $n \geq+2$, we expect our analysis to be over-conservative, and it should be possible to derive stronger bounds from a dedicated analysis. This situation is analogous to that found for warm dark matter (WDM), where a simple likelihood analysis of the type we used [45] finds significantly weaker constraints than those obtained in dedicated simulations [46].

\section{BARYON-DM INTERACTIONS AND SUPPRESSION OF SMALL-SCALE STRUCTURE}

Baryon-DM interactions may affect small-scale structure, and galactic substructure, in a number of ways. The most straightforward effect is to suppress the growth in the early Universe of small-scale power and thus the halo mass function at the low-mass end. Here we estimate the effect compatible with our constraints. We then, in the Section 
that follows, discuss the possible consequences for the evolution of galaxies at late times.

We compute the halo mass function using the extended Press-Schechter formalism [47 49],

$$
\frac{d n_{h}}{d M}(M)=\frac{\rho_{m}}{M}\left|\frac{d \sigma}{d M}\right| f\left(\delta_{\chi}(z), \hat{\sigma}\right)
$$

where $\rho_{m}$ is the mean matter density in the Universe, and $\hat{\sigma}$ is the variance,

$$
\hat{\sigma}(M)=\int \frac{d k}{k} \Delta_{m}(k)|W(k, R)|^{2}
$$

Here $\Delta_{m}(k)$ is the matter density variance and $W(k, R)$ is a tophat window function of radius $R$, corresponding to a halo of mass $M=\left(4 \pi R^{3} \rho_{m} / 3\right)$. For the function $f$ we use the Sheth-Tormen functional form [50], given by

$$
f\left(\delta_{\chi}, \hat{\sigma}\right)=A \frac{\nu}{\hat{\sigma}} \sqrt{\frac{a}{2 \pi}}\left[1+\frac{1}{\left(a \nu^{2}\right)^{q}}\right] e^{-a \nu^{2} / 2}
$$

where $\nu=(\delta / \sqrt{\hat{\sigma}}), a=0.75, q=0.3, A=0.322$, and $\delta=0.686$ is the critical density of collapse.

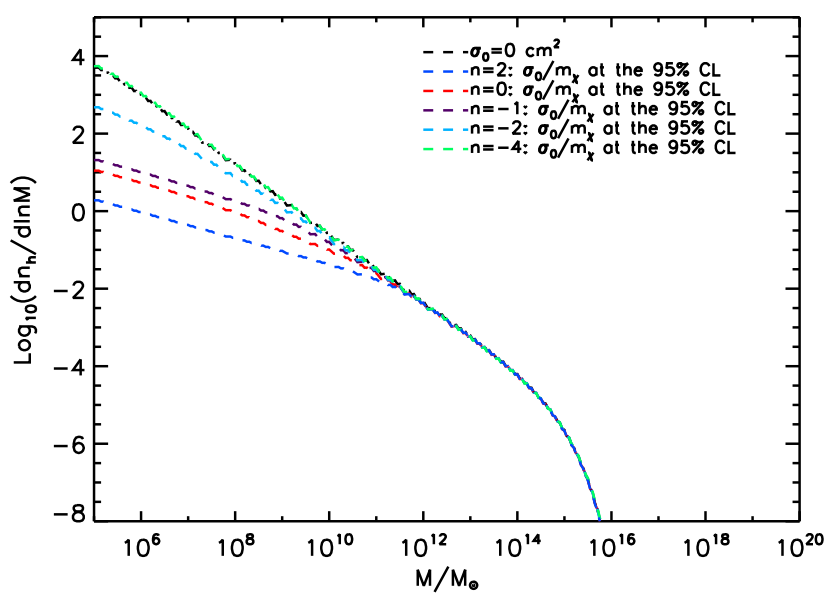

FIG. 5: Halo mass function as a function of mass. A model with no scattering is shown in black/dashed lines, and models with different velocity-dependent cross sections are shown with a value of $\sigma_{0} / m_{\chi}$ taken at the $95 \%$ CL limit from the analysis with CMB and Lyman- $\alpha$ data in Table I

Fig. 5 shows the halo mass function as a function of mass for a model with no scattering (black/dashed line) along with models with different velocity-dependent cross sections with a value of $\left(\sigma_{0} / m_{\chi}\right)$ taken at the $95 \%$ CL limit from Tab. [1] using CMB + Lyman- $\alpha$ data. In all cases, the cosmological parameters were fixed to the best fit point at the given value of $\left(\sigma_{0} / m_{\chi}\right)$.

Two main lessons can be drawn from Fig. 5. First, the combined constraints from linear cosmology imply that DM-baryon scattering cannot affect the halo mass function for structures more massive than $\sim 10^{12} M_{\odot}$. This result is model-independent. It simply reflects the scale at which the observational LSS constraint is applied in our analysis, $k \sim 1 \mathrm{Mpc}^{-1}$, since

$$
M=\frac{4 \pi}{3}\left(\frac{\pi}{k}\right)^{3} \rho_{m} \sim 2 \times 10^{12} M_{\odot}\left(\frac{k}{1 \mathrm{Mpc}^{-1}}\right)^{-3}
$$

For smaller mass halos, significant suppression of structure is in principle possible.

Second, note that the model with $n=-4$ (scattering cross section scaling as $v^{-4}$ ) does not have any effect on the halo mass function. This occurs because models with $n<-3$ have the feature that they freeze-out towards high redshift, when the collision velocities (governed by thermal motion) get large, and freeze-in at lower redshift when the velocities drop. In contrast, models with $n>-3$ are initially important and then subsequently freeze-out as the Universe expands and cools. As halos of smaller mass form earlier, only modes of $n>-3$ can affect the primordial halo mass function on small scales while still satisfying CMB/LSS constraints that are only directly sensitive to $z \lesssim 10^{6}$. 
To conclude this Section, Fig. 5 teaches us that DM-baryon scattering can affect the halo mass function on small scales, but this effect is purely a memory effect from early (linear) times (high redshift). This is a useful lesson. It means that N-body simulations aiming to study the effect of scattering, need not incorporate the scattering explicitly. Instead, ordinary collisionless codes should be applicable, where a modified linear matter power spectrum, as we computed here, is used as input to encode the effect of scattering.

Finally, we comment that the analysis of the halo mass function above is not sufficient by itself to expose highly nonlinear details within small scale objects, such as the presence or absence of central cusps etc. We briefly discuss constraints at this level of detail in Sec. VI.

\section{LATE-TIME EFFECTS OF BARYON-DM INTERACTIONS}

In Sec.VWwe estimated the maximal effect of DM-baryon scattering on the primordial halo mass function. The effects discussed in that section encoded early time dynamics, for which our linear analysis was adequate. Here, in contrast, we comment briefly on the implications of our constraints for the late-time, non-linear evolution of galaxies. Galaxies are complicated objects, and the models for their detailed structure contain considerable theoretical uncertainty (see e.g. 51]). Still, in some generic cases of interest it is straightforward to see that our results strongly constrain the effect that DM-baryon scattering could have on the late-time evolution of galaxies.

A clear example pertains to the halos of galaxies like our own Milky Way. The mean free path of a hydrogen atom, traversing a typical galaxy like our Milky Way, to elastically scatter from a DM particle is

$$
\lambda \sim 0.5\left(\frac{\bar{\sigma} / m_{\chi}}{\mathrm{cm}^{2} / \mathrm{g}}\right)^{-1}\left(\frac{\rho_{\chi}}{0.4 \mathrm{GeV} / \mathrm{cm}^{3}}\right)^{-1} \mathrm{Mpc},
$$

where we have used a density $\rho_{\chi} \simeq 0.4 \mathrm{GeV} / \mathrm{cm}^{3}$ characteristic of the Milky Way halo at the location of the Solar System, and $\bar{\sigma}$ is evaluated for a velocity $v \sim 300 \mathrm{~km} \mathrm{~s}^{-1} \sim 10^{-3}$, the virial velocity in the Milky Way. The canonical numerical value $\bar{\sigma} \simeq 1 \mathrm{~cm}^{2} \mathrm{~g}^{-1}$ is chosen having in mind the distance traveled by a particle moving at $v \simeq 300 \mathrm{~km} \mathrm{~s}$

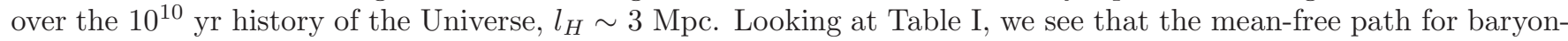
DM scattering is constrained by CMB/Lyman- $\alpha$ data to be far larger than the distance a particle travels through a halo in the history of the Universe: $\left(\lambda / l_{H}\right) \gg 1$, for any $n$.

This estimate is valid for a MW-type object at the solar cycle. To extend this estimate to larger or smaller objects like galaxy clusters and dwarf galaxies, we write

$$
\frac{\lambda}{l_{H}}>30\left(\frac{\lambda_{\text {Tab.I }(\mathrm{n})}}{100 \mathrm{Mpc}}\right)\left(\frac{v}{10^{-3}}\right)^{-n-1}\left(\frac{\rho_{\chi}}{0.4 \mathrm{GeV} / \mathrm{cm}^{3}}\right)^{-1},
$$

where for $\lambda_{\text {Tab.I(n) }}$ we use the result on the last column of Tab. I and for $v$ and $\rho_{\chi}$ we use the characteristic values for the object of interest. We learn that $n>+2$ is needed to affect structure on galaxy cluster scales, with $\rho_{\chi}$ smaller by

some two orders of magnitude and $v$ larger by a factor of ten or so compared to the MW halo. In contrast, $n<-4$ is needed to affect dwarf galaxy scales, with $v \sim 10^{-4}$.

\section{CONCLUSIONS}

We have provided a model-independent analysis of the constraints imposed to baryon-DM interactions by CMB data from Planck and Lyman- $\alpha$-forest data from the SDSS. Our work extended and improved upon earlier analyses for specific models like DM millicharge, electromagnetic dipole moments, and velocity-independent cross section (see App. C). We found that the Lyman- $\alpha$-forest data, included here for the first time in this context, considerably strengthen the constraints beyond the reach of the CMB alone. We highlighted the interplay between bulk and thermal velocities, pointed out to the cross-over between them at $z \sim 10^{4}$, and suggested an approximate way to take it into account.

DM-baryon scattering cannot affect the halo mass function for $M>10^{12} M_{\odot}$. There is still room, after the new constraints are imposed, for a potentially consequential suppression of primordial power on smaller, subgalactic scales. In the halos of galaxies like the Milky Way, outside of the innermost $1 \mathrm{kpc}$, our constraints imply that the baryon-DM interaction rate is, regardless of the model, too small to affect the distribution of matter at late times.

Our observation that bulk DM-baryon velocities become greater than thermal velocities at redshifts $z \simeq 10^{4}$ may have interesting consequences. It suggests that (unless the cross-section power-law index is $n=-1$ ) the baryon-DM drag does not vary linearly with the relative velocity. If so, then the Boltzmann equations for the evolution of baryons 
and dark matter become nonlinear. This has two consequences: First, the evolution of each Fourier mode cannot be described by the standard linear equations; and second, different Fourier modes become coupled, thus inducing non-Gaussianity. In this paper, we have included these effects in a mean-field approach. In this treatment, each Fourier mode is evolved independently, and the coupling to other Fourier modes is taken into account by augmenting the thermal velocity dispersion with a dispersion due to bulk velocities. While this approach should be fairly accurate and provide conservative quantitative constraints, it will be interesting in future work to quantify these effects more precisely and to investigate the implications of the non-Gaussianity that a baryon-DM interaction may induce.

\section{Acknowledgments}

We thank Simeon Bird, Jo Bovy, Raphael Flauger, Anze Slosar and Matias Zaldarriaga for useful discussions. We are particularly grateful to Uroš Seljak for suggesting the use of the Lyman- $\alpha$ forest data. CD was supported by the National Science Foundation grant number AST-0807444, NSF grant number PHY-088855425, and the Raymond and Beverly Sackler Funds. KB was supported by DOE grant de-sc0009988. MK was supported by NASA NNX12AE86G, NSF 0244990, and the John Templeton Foundation.

\section{Appendix A: Thermalization}

The thermalization rate is calculated similarly to the momentum exchange rate. The change in energy of a DM particle per collision is

$$
\Delta \epsilon_{\chi}=\left(\frac{\vec{p}_{\chi}+\vec{p}_{b}}{m_{\chi}+m_{b}}\right) \cdot \Delta \vec{p}_{\chi}=\vec{v}_{c m} \cdot \Delta \vec{p}_{\chi}
$$

where $\vec{v}_{c m}$ is the boost velocity to the center of mass frame of the collision. Focusing on the limit where peculiar velocities are smaller than the velocity dispersion, we can set the peculiar velocity to zero. The specific heating rate is then

$$
\begin{aligned}
\frac{d Q_{\chi}}{d t}= & \frac{m_{\chi} \rho_{b}}{m_{\chi}+m_{b}} \int \frac{d \hat{n}_{b}}{4 \pi} \int d v_{b} v_{b}^{2} f_{b}\left(v_{b}\right) \int \frac{d \hat{n}_{\chi}}{4 \pi} \int d v_{\chi} v_{\chi}^{2} f_{\chi}\left(v_{\chi}\right) \int d \hat{n}\left(\frac{d \sigma\left(\left|\vec{v}_{\chi}-\vec{v}_{b}\right|\right)}{d \hat{n}}\right)\left|\vec{v}_{\chi}-\vec{v}_{b}\right|^{2} \\
& \times\left(\hat{n}-\frac{\vec{v}_{\chi}-\vec{v}_{b}}{\left|\vec{v}_{\chi}-\vec{v}_{b}\right|}\right) \cdot\left(\frac{m_{\chi} \vec{v}_{\chi}+m_{b} \vec{v}_{b}}{m_{\chi}+m_{b}}\right) \\
= & -\frac{2^{\frac{n+5}{2}} \Gamma\left(3+\frac{n}{2}\right)}{\sqrt{\pi}} \frac{a m_{\chi} \rho_{b} \sigma_{0}}{\left(m_{\chi}+m_{b}\right)^{2}}\left(\frac{T_{b}}{m_{b}}+\frac{T_{\chi}}{m_{\chi}}\right)^{\frac{n+1}{2}}\left(T_{\chi}-T_{b}\right) .
\end{aligned}
$$

From this, neglecting the time derivative of the mean baryonic molecular weight, we obtain Eq. (13).

\section{Appendix B: Peculiar velocity beyond leading order}

The exact solution for the peculiar velocity $V_{\chi}$ is given by

$$
\frac{d \vec{V}_{\chi}}{d t}=-\vec{V}_{\chi} \frac{{ }_{n} \rho_{b} \sigma_{0}\left(\frac{\left\langle(\Delta \vec{v})^{2}\right\rangle}{3}\right)^{\frac{n+1}{2}}}{m_{\chi}+m_{b}} \mathcal{G}_{n}\left(\frac{3 \vec{V}_{\chi}^{2}}{\left\langle(\Delta \vec{v})^{2}\right\rangle}\right),
$$

in real space, with the dimensionless function:

$$
\mathcal{G}_{n}(w)=1+\frac{n+1}{10} w+\frac{(n+1)(n-1)}{280} w^{2}+\frac{(n+1)(n-1)(n-3)}{15120} w^{3}+\cdots .
$$

\section{Appendix C: Comparison to previous work and specific dark matter-baryon interaction models}

Constraints on DM-baryon scattering were derived in previous work for some specific particle physics models. Here we compare our results with existing bounds. 


\section{Velocity-independent scattering}

Cosmological constraints on a velocity-independent scattering cross section were derived in Ref. [14]. We generally agree with the derivation in 14], besides from an $\mathcal{O}(1)$ numerical difference in the expression for the momentum transfer rate $R_{\chi}$ and, again, from the neglect of peculiar vs. thermal velocities at low redshift. Our CMB+Lyman- $\alpha$ constraint in Tab. [ improves on the bound of Ref. [14] by about two orders of magnitude, most likely due to the incorporation of Lyman- $\alpha$ data in our analysis.

\section{DM millicharge}

If DM carries a millicharge $q_{\chi}=\epsilon e$, where $e$ is the electron charge and $\epsilon \ll 1$, then photon exchange with protons induces a cross section

$$
\frac{d \sigma}{d c_{\theta}}=\left(\frac{m_{\chi}+m_{H}}{m_{\chi} m_{H}}\right)^{2} \frac{2 \pi \epsilon^{2} \alpha^{2}}{v^{4}\left(1-c_{\theta}\right)^{2}}
$$

We focus here on $m_{\chi} \gg m_{H}$. The scattering on helium is suppressed by the reduced mass, $\sigma_{H e}=\sigma_{0} / 4$, leading to $\mathcal{F}_{\mathrm{He}}=0.82$ when all of the helium and hydrogen are ionized. Helium recombination begins around $z \sim 6 \cdot 10^{3}$ and completes by $z \sim 2 \cdot 10^{3}$; it is straightforward to include helium recombination in the momentum transfer equations, but for simplicity we avoid this complication and simply set $\mathcal{F}_{\mathrm{He}}=0.76$, neglecting DM-helium scattering altogether. Note that scattering on electrons is negligible for the momentum exchange, as it amounts to $R_{\chi} \rightarrow$ $R_{\chi}\left[1+x_{e}\left(1-f_{H e}\right)\left(m_{e} / m_{H}\right)^{\frac{1}{2}}\right]$, where $x_{e}=n_{e} / n_{H}$ is the free electron fraction and $m_{e}=5.44 \cdot 10^{-4} m_{H}$ is the electron mass.

The forward divergence in Eq. (C1) is regulated by Debye screening due to free electrons in the plasma [18], implying a minimum scattering angle $\theta_{\text {min }} \approx 2 \epsilon \alpha /\left(3 T \lambda_{D}\right)$, with $\lambda_{D}=\sqrt{T /\left(4 \pi \alpha n_{e}\right)}$. This gives a momentum exchange cross section

$$
\bar{\sigma}(v) \approx\left(\frac{m_{\chi}+m_{H}}{m_{\chi} m_{H}}\right)^{2} \frac{2 \pi \epsilon^{2} \alpha^{2}}{v^{4}} \ln \left(\frac{9 T^{3}}{4 \pi \epsilon^{2} \alpha^{3} x_{e} n_{H}}\right)
$$

or in our notation (taking $m_{\chi} \gg m_{H}$, and measuring velocity in units of $c$ ),

$$
\begin{aligned}
n & =-4, \\
\sigma_{0} & \approx 9.6 \times 10^{-42}\left(\frac{\epsilon}{10^{-6}}\right)^{2}\left[1-0.03 \ln \left(\frac{\epsilon}{10^{-6}}\right)\right] \mathrm{cm}^{2} .
\end{aligned}
$$

This expression is valid for $z>1100$ or so, when $x_{e} \approx 1$. Upon recombination, $x_{e}$ falls quickly below unity $\left[x_{e}(z \sim\right.$ 1000) $\left.\sim 10^{-3}\right]$, increasing the $\ln \epsilon$ correction to $\ln \left(\epsilon \sqrt{x_{e}}\right)$. For the very small values of $\epsilon$ that we find here, this change in the logarithm makes no significant difference to the results. Furthermore, accounting for recombination amounts to scaling

$$
R_{\chi} \rightarrow \min \left(x_{e}, 1\right) \times R_{\chi}
$$

so that scattering via DM millicharge halts anyway once protons combine into neutral hydrogen.

Using the 95\%CL bound for $n=-4$ from Tab. I] we find

$$
\epsilon<1.8 \cdot 10^{-6}\left(\frac{m_{\chi}}{\mathrm{GeV}}\right)^{\frac{1}{2}}
$$

Cosmological constraints on DM millicharge were derived in [17 20]. Our numerical result for the 95\%CL limit is stronger by a factor of two than the bound derived in Ref. [18]. However, there are conceptual differences between our analysis and the one in 18]. Notably, we here computed the rate of momentum transfer in linear theory, while the bound derived in Ref. [18] was based on the rough argument of imposing kinetic decoupling at recombination, and ignored the bulk velocity altogether. As a result, the momentum transfer rate as defined in Ref. [18] is not the proper quantity for linear theory. In addition, as discussed in Sec. IIB at redshift $z<10^{4}$ the typical peculiar velocity itself becomes large compared with the thermal motion. This regulates the low-velocity enhancement $\left(\propto v^{-4}\right)$ of the millicharge interaction. As mentioned in Sec. IV had we ignored this effect and considered only the thermal motion (as was done in Ref. [18]) we would have found a bound on $\epsilon$ that would be stronger by a factor of $\sim 3$ compared to Eq. (C5). 


\section{DM electric and magnetic dipole moment}

DM magnetic and electric dipole moment (MDM and EDM) was considered in Ref. [15], expressed as

$$
\mathcal{L}=-\frac{i}{2} \bar{\chi} \sigma_{\mu \nu}\left(\mathcal{M}+\gamma_{5} \mathcal{D}\right) \chi F^{\mu \nu}
$$

where the MDM is $\mathcal{M}$ and the EDM is $\mathcal{D}$.

We agree with the cosmological calculation in [15]. The momentum transfer cross section for DM-proton collisions is

$$
\bar{\sigma}=3 \alpha \mathcal{M}^{2}\left[1-\frac{m_{H}\left(m_{H}+4 m_{\chi}\right)}{3\left(m_{H}+m_{\chi}\right)^{2}}\right],
$$

for MDM, corresponding to $n=0$ in our notation; and

$$
\bar{\sigma}=\frac{2 \alpha \mathcal{D}^{2}}{v^{2}}
$$

for EDM, corresponding to $n=-2$. Assuming $m_{\chi} \gg m_{H}$, ignoring helium again, and using Tab. \we find at 95\%CL:

$$
\begin{aligned}
& \mathcal{M}<1.7 \times 10^{-12} \text { e cm } \\
& \mathcal{D}<9.2 \times 10^{-16}\left(\frac{m_{\chi}}{1 \mathrm{GeV}}\right)^{\frac{1}{2}} \text { e cm } .
\end{aligned}
$$

The $\operatorname{EDM}(\mathcal{D})$ bound we find is stronger by about a factor of two than that reported in [15] for CMB/LSS. For $\operatorname{MDM}(\mathcal{M})$, the original analysis of [15] does not apply, and our numerical result is new.

[1] G. Jungman, M. Kamionkowski and K. Griest, Phys. Rept. 267, 195 (1996) arXiv:hep-ph/9506380.

[2] L. Bergstrom, Rept. Prog. Phys. 63, 793 (2000) arXiv:hep-ph/0002126].

[3] G. Bertone, D. Hooper and J. Silk, Phys. Rept. 405, 279 (2005) arXiv:hep-ph/0404175.

[4] G. D'Amico, M. Kamionkowski and K. Sigurdson, arXiv:0907.1912 [astro-ph.CO].

[5] A. Loeb and M. Zaldarriaga, Phys. Rev. D 71, 103520 (2005) astro-ph/0504112.

[6] D. E. Kaplan, M. A. Luty and K. M. Zurek, Phys. Rev. D 79, 115016 (2009) [arXiv:0901.4117 [hep-ph]].

[7] K. M. Zurek, arXiv:1308.0338 [hep-ph].

[8] B. Moore, S. Ghigna, F. Governato, G. Lake, T. R. Quinn and J. Stadel, arXiv:astro-ph/9711259

[9] M. Kamionkowski and A. R. Liddle, Phys. Rev. Lett. 84, 4525 (2000) arXiv:astro-ph/9911103.

[10] J. S. Bullock, arXiv:1009.4505 [astro-ph.CO].

[11] D. H. Weinberg, J. S. Bullock, F. Governato, R. K. de Naray and A. H. G. Peter, arXiv:1306.0913 [astro-ph.CO].

[12] B. Famaey and S. McGaugh, J. Phys. Conf. Ser. 437, 012001 (2013) arXiv:1301.0623 [astro-ph.CO]].

[13] P. J. E. Peebles, arXiv:1305.6859 [astro-ph.CO].

[14] X. l. Chen, S. Hannestad and R. J. Scherrer, Phys. Rev. D 65, 123515 (2002) arXiv:astro-ph/0202496.

[15] K. Sigurdson, M. Doran, A. Kurylov, R. R. Caldwell and M. Kamionkowski, Phys. Rev. D 70, 083501 (2004) [Erratum-ibid. D 73, 089903 (2006)] arXiv:astro-ph/0406355.

[16] C. Boehm and R. Schaeffer, arXiv:astro-ph/0410591.

[17] A. D. Dolgov, S. L. Dubovsky, G. I. Rubtsov and I. I. Tkachev, arXiv:1310.2376 [hep-ph].

[18] S. D. McDermott, H. -B. Yu and K. M. Zurek, Phys. Rev. D 83, 063509 (2011) arXiv:1011.2907 [hep-ph]].

[19] S. L. Dubovsky and D. S. Gorbunov, Phys. Rev. D 64, 123503 (2001) arXiv:astro-ph/0103122.

[20] S. L. Dubovsky, D. S. Gorbunov and G. I. Rubtsov, JETP Lett. 79, 1 (2004) [Pisma Zh. Eksp. Teor. Fiz. 79, 3 (2004)] arXiv:hep-ph/0311189.

[21] S. Davidson, S. Hannestad and G. Raffelt, JHEP 0005, 003 (2000) hep-ph/0001179.

[22] L. Chuzhoy and A. Nusser, Astrophys. J. 645, 950 (2006) arXiv:astro-ph/0408184.

[23] J. Hu and Y. Q. Lou, arXiv:0711.3555 [astro-ph].

[24] G. D. Starkman, A. Gould, R. Esmailzadeh and S. Dimopoulos, Phys. Rev. D 41, 3594 (1990).

[25] A. L. Erickcek, P. J. Steinhardt, D. McCammon and P. C. McGuire, Phys. Rev. D 76, 042007 (2007) arXiv:0704.0794 [astro-ph]].

[26] I. F. M. Albuquerque and L. Baudis, Phys. Rev. Lett. 90, 221301 (2003) [Erratum-ibid. 91, 229903 (2003)] astro-ph/0301188.

[27] V. Sanglard et al. [EDELWEISS Collaboration], Phys. Rev. D 71 (2005) 122002 astro-ph/0503265.

[28] D. S. Akerib et al. [CDMS Collaboration], Phys. Rev. D 73, 011102 (2006) astro-ph/0509269. 
[29] D. S. Akerib et al. [CDMS Collaboration], Phys. Rev. Lett. 96, 011302 (2006) astro-ph/0509259.

[30] P. Natarajan, A. Loeb, J. -P. Kneib and I. Smail, Astrophys. J. 580, L17 (2002) astro-ph/0207045].

[31] B. Qin and X. -P. Wu, Phys. Rev. Lett. 87 (2001) 061301 astro-ph/0106458.

[32] R. N. Mohapatra and V. L. Teplitz, Phys. Rev. Lett. 81, 3079 (1998) hep-ph/9804420].

[33] R. H. Cyburt, B. D. Fields, V. Pavlidou and B. D. Wandelt, Phys. Rev. D 65, 123503 (2002) arXiv:astro-ph/0203240.

[34] B. D. Wandelt, R. Dave, G. R. Farrar, P. C. McGuire, D. N. Spergel and P. J. Steinhardt, astro-ph/0006344.

[35] G. D. Mack, J. F. Beacom and G. Bertone, Phys. Rev. D 76, 043523 (2007) arXiv:0705.4298 [astro-ph]].

[36] G. D. Mack and A. Manohar, arXiv:1211.1951 [astro-ph.CO].

[37] D. N. Spergel and P. J. Steinhardt, Phys. Rev. Lett. 84, 3760 (2000) astro-ph/9909386].

[38] C. P. Ma and E. Bertschinger, Astrophys. J. 455, 7 (1995) arXiv:astro-ph/9506072.

[39] D. Tseliakhovich and C. Hirata, Phys. Rev. D 82, 083520 (2010) arXiv:1005.2416 [astro-ph.CO]].

[40] A. Lewis and S. Bridle, Phys. Rev. D 66, 103511 (2002) arXiv:astro-ph/0205436.

[41] P. A. R. Ade et al. [Planck Collaboration], arXiv:1303.5075 [astro-ph.CO].

[42] P. McDonald et al. [SDSS Collaboration], Astrophys. J. 635, 761 (2005) arXiv:astro-ph/0407377.

[43] J. Dunkley, E. Calabrese, J. Sievers, G. E. Addison, N. Battaglia, E. S. Battistelli, J. R. Bond and S. Das et al., arXiv:1301.0776 [astro-ph.CO].

[44] Z. Hou, C. L. Reichardt, K. T. Story, B. Follin, R. Keisler, K. A. Aird, B. A. Benson and L. E. Bleem et al., arXiv:1212.6267 [astro-ph.CO].

[45] K. Abazajian, Phys. Rev. D 73, 063513 (2006) astro-ph/0512631].

[46] U. Seljak, A. Makarov, P. McDonald and H. Trac, Phys. Rev. Lett. 97, 191303 (2006) astro-ph/0602430.

[47] J. R. Bond, S. Cole, G. Efstathiou and N. Kaiser, Astrophys. J. 379, 440 (1991).

[48] R. G. Bower, Mon. Not. Roy. Astron. Soc. 248, 332 (1991).

[49] C. G. Lacey and S. Cole, Mon. Not. Roy. Astron. Soc. 262, 627 (1993).

[50] R. K. Sheth and G. Tormen, Mon. Not. Roy. Astron. Soc. 329 (2002) 61 astro-ph/0105113.

[51] S. Trujillo-Gomez, A. Klypin, J. Primack and A. J. Romanowsky, Astrophys. J. 742, 16 (2011) arXiv:1005.1289 [astroph.CO]]. 